\title{
Research on PID control and Simulation with M Language of Matlab Program
}

\author{
Yuqiang Jin ${ }^{1}$, Yuliang Chen ${ }^{2}$, Zijian Lin $^{3}$ and Junwei Lei ${ }^{3, a}$ \\ ${ }^{1}$ Department of Training, Naval aeronautical and astronautical University, Yanti, 264001 \\ ${ }^{2}$ Department of Ordnance Science and Technology, Naval aeronautical and astronautical \\ University Yantai, China \\ ${ }^{3}$ Department of control engineering, Naval aeronautical and astronautical University
}

Yanti, 264001

aleijunwei@126.com

Keyword: PID, Stability, Robustness, Simulation, Program

\begin{abstract}
In this paper, we use the M language of Matlab software to study the programming and implementation of PID control for a class of simple first order systems. According to the proportional, proportional integral and full proportional integral differential control three cases, We separately conduct the procedure compilation , give the corresponding simulation results, finally carry on the contrast analysis and illustrate the characteristics of three basic ways to control their own.
\end{abstract}

\section{Introduction}

PID control is a simple and good control method so that in the industrial control field it has been applied widely. Now most of the missiles and unmanned aerial vehicles as well as the use of multipurpose robots in the control loop are used in the PID structure. In the modern vehicle, PID control plays an important role mainly because it has a strong ability to adapt and the controller which works in accordance with it has been industrialized production, so that it can be widely used in daily life and its technology is increasingly perfect[7].Although the PID control algorithm is simple, its programming implementation issues can be flexible. What's more, engineers propose a variety of improved PID control algorithm based on the basic PID control algorithm, and even have been sorted into related books published. Therefore, PID control algorithm of engineering experiment is still an open field, and there are still new achievements in the field. Based on the above reasons, This paper studies the PID control of the Matlab software $M$ language implementation issues.

\section{PID Control Theory}

PID controller is a linear controller[6], which constitutes the control deviation based on the given value and the actual output value.

$$
e(t)=r(t)-u(t)
$$

The control law of PID is:

$$
u(t)=k_{p}\left(e(t)+\frac{1}{T_{I}} \int_{0}^{t} e(t) d t+\frac{T_{d} e(t)}{d t}\right)
$$

Written in the form of transfer function:

$$
G(s)=\frac{U(s)}{E(s)}=k_{p}\left(1+\frac{1}{T_{i} s}+T_{d} s\right)
$$

In the formula:

$K_{p}$-Proportional coefficient; 
$T_{i}$ - Integral time constant;

$T_{d}$ —Differential time constant.

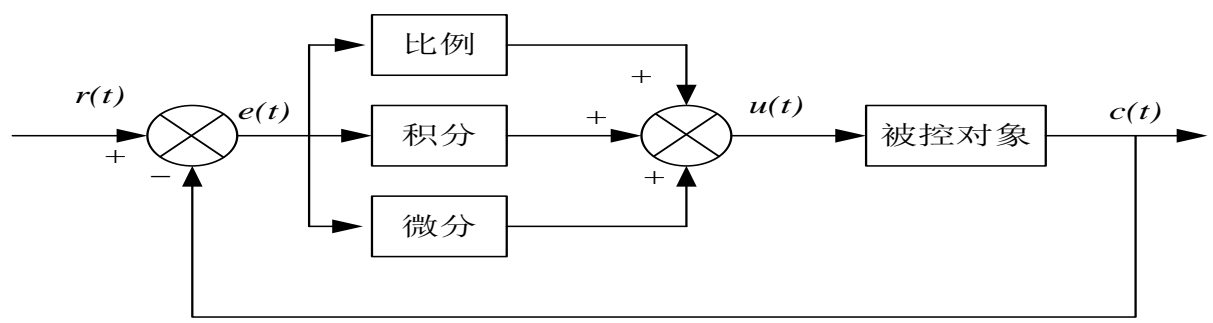

Fig. 1 PID control system structure diagram

As shown in figure PID control system of the structure, the system is composed of PID controller and the controlled object two parts. And the control signal is generated by the PID controller so as to control the object.PID controller consists of the ratio (P), the integral (I) and the differential (D) three parts. We can adjust three parameters $\left(k_{p}, k_{i}, k_{d}\right)$ from the formula (3.4) to achieve the best results. However, in many cases, you can achieve the best results by adjusting one or two parameters instead of each parameter. But in general, the proportion of control is indispensable.

Using M software MATLAB language and PID control principle, I do the following several simple simulation programs, and get the simulation results.

\section{Simulation of P Control and PI Control}

Here we design the time interval is $0.001 \mathrm{~s}$, the simulation time is $10 \mathrm{~s}$, the initial value is 0 and the expected value is 1 .Then we make $x \rightarrow x^{d}$ through the program design and firstly design the proportional control, that is, $\mathrm{u}=\mathrm{k} 1 * \mathrm{e}$. Programming is as follows:

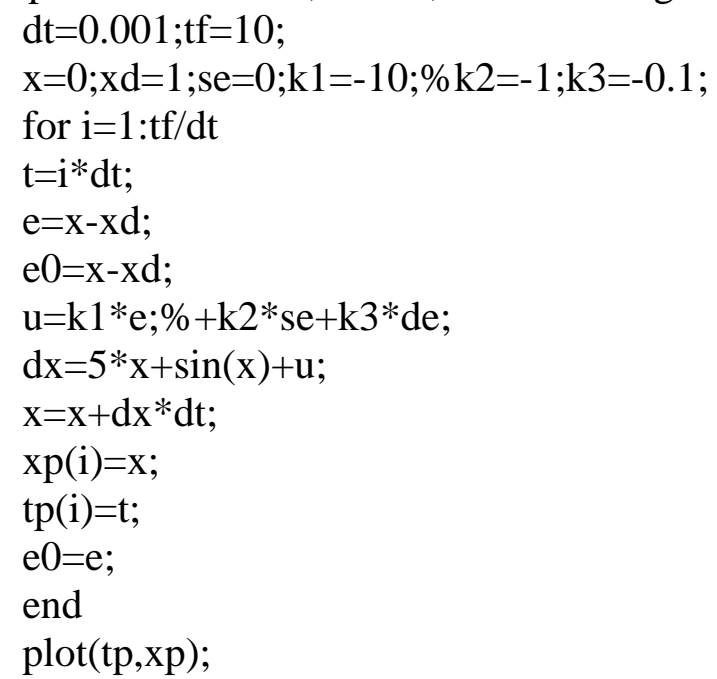

Put the program into the MATLAB, and the simulation is shown as Fig.2.

As can be seen from the diagram, essentially, the proportional controller is a amplifier with adjustable gain through the proportional control program design. Adding controller gain $\mathrm{Kp}$ in the program can improve the open-loop gain of the system and reduce the steady state error of the system so as to improve the control precision of the system. Through the simulation diagram, we can get that adding proportional control in the program design does not achieve the desired effect and the value of $\mathrm{X}$ does not reach the desired value. It still exists the system static error, so we change the design process again, which adds the integral control on the original, that is, $\mathrm{u}=\mathrm{k} 1 * \mathrm{e}+\mathrm{k} 2 * \mathrm{se}$.

The simulation program is

$\mathrm{dt}=0.001 ; \mathrm{tf}=10$; 


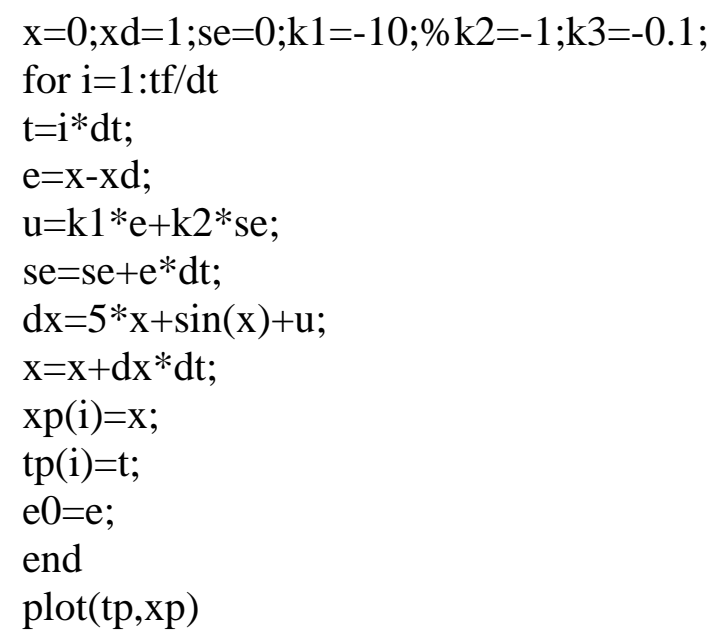

And the simulation is shown as Fig.3.

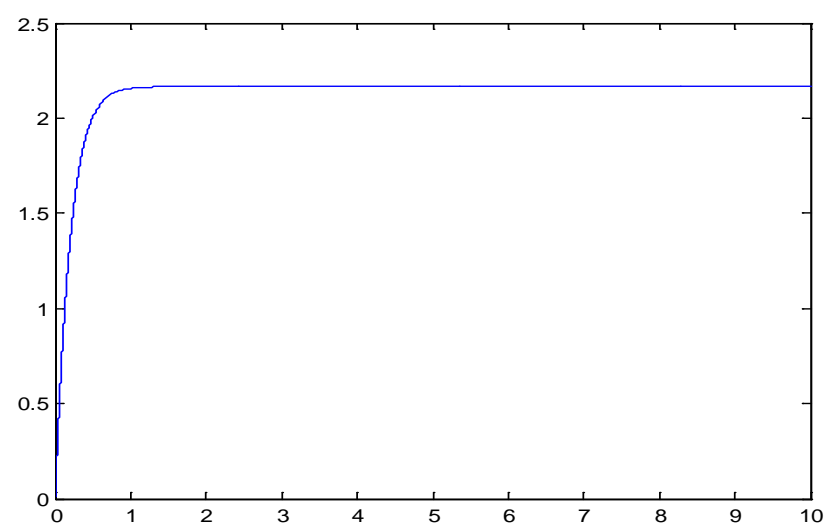

Fig. 2 Proportional control simulation diagram

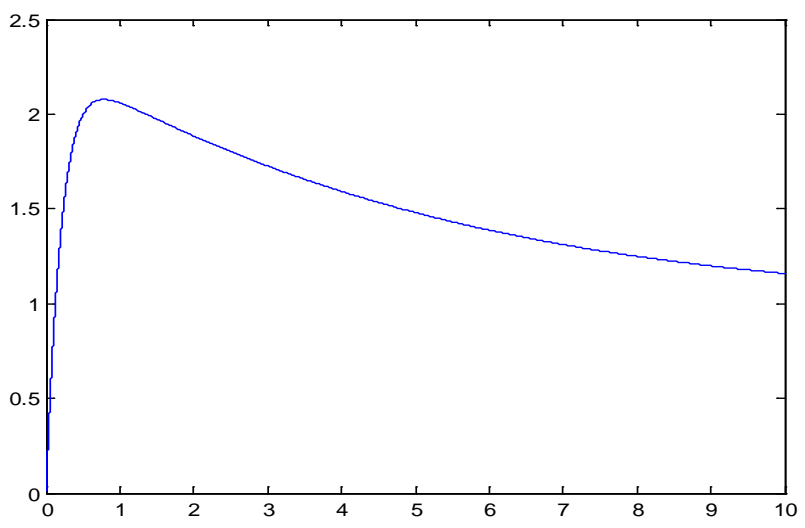

Fig. 3 Proportional integral control simulation

It can be found from the above, through the PI control law design, the value of $\mathrm{X}$ can be close to the expected value in about 10s. So in the back of the simulation design, we can take this way to simulate. In summary, the integral link can effectively improve the static performance of the system, but can also destroy the stability of the system quickly. PI control, however, integrates the advantages and disadvantages of the proportion and integration, which can not only eliminate the interference but also be used to eliminate the static error.

\section{Simulation of PID Control}

According to the first two procedures, we see that without complete PID control, the results of the simulation diagram is not very ideal. So we use the complete PID control to make the simulation curve of $\dot{x}=5 x+\sin x+u$.Suppose the simulation time is $15 \mathrm{~s}$, the time interval is $0.001 \mathrm{~s}$, the initial 
value is 0 ,the expected value is 1 and the control law is $\mathrm{u}=\mathrm{k} 1 * \mathrm{e}+\mathrm{k} 2 * \mathrm{se}+\mathrm{k} 3 * \mathrm{de}$. Therefore the simulation program is as follows:

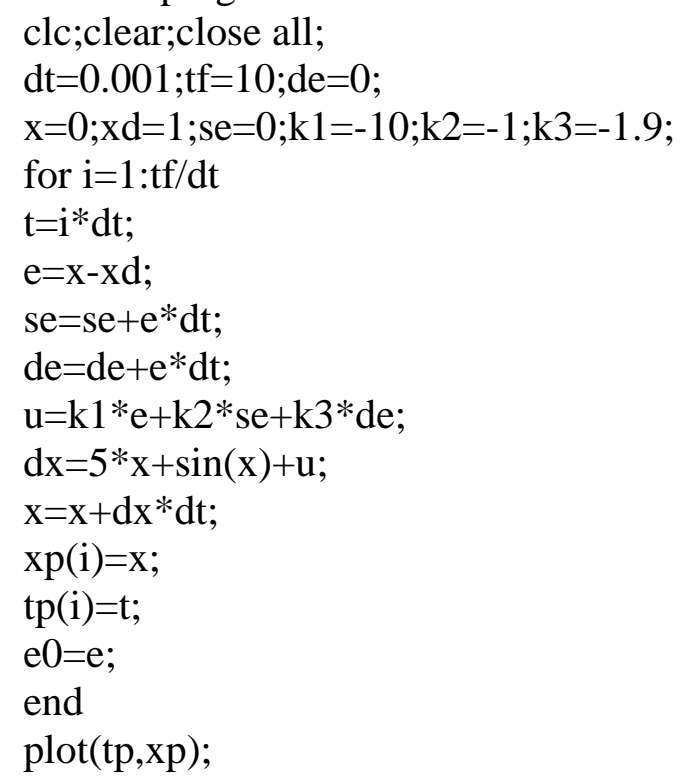

In the process of programming, transform the differential coefficient K3.Take $\mathrm{k} 3=0.1$ and $\mathrm{k} 3=1.9$, respectively, get the following two simulation images:

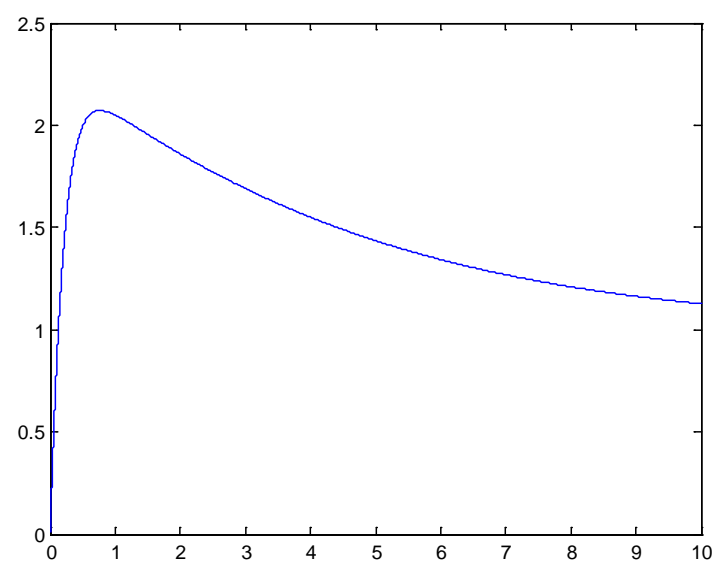

Fig. 4 k3=0.1

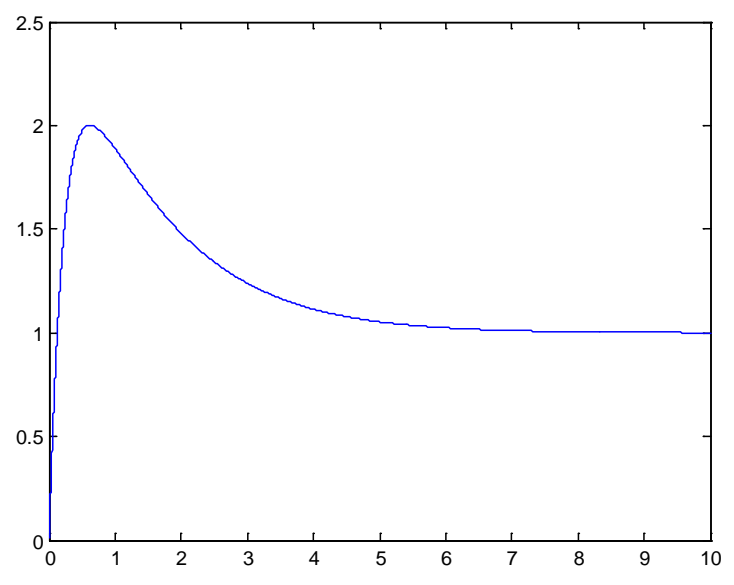

Fig. 5 k3=1.9

Through the above simulation results, it can be seen that the complete PID control can eliminate the static error and have a good speed.

\section{Conclusions}

In this paper, we mainly study the problem of computer programming for three basic PID control strategies, which are proportional control, proportional integral control and proportional integral derivative control. Controlled object is selected as a kind of basic first order system, and the simulation language uses $M$ software Matlab language. Finally we give the corresponding simulation results, and compare and analyze the response curves.

\section{References}

[1]Yokomaku Y. The Stratospheric Platform Airship R\&D Program of Japan. The 2nd Stratospheric Platform Systems Workshop, Tokyo Japan.2000:7 13

[2]S.P. Jones, J.D. Laurier. Aerodynamic Estimation Techniques for Aerostats and Airships. AIAA Lighter-than-Air Systems Conference,Annapolis,2004: 88 94

[3]M.T. Soylemeza, N. Munrob, H. Bakic. Fast Calculation of Stabilizing PID Controllers. 
Automatic, 2 003,39(7):121 126

[4]Etkin B, Theory of the flight of Airplanes in Isotropic Turbulence Review an Extension, AGARD Rept.1961:372

[5] F. Sun, Y. Zhao, T. Zhou, Identify fully uncertain parameters and design controllers based on synchronization, Chaos, Solitons and Fractals, 34, (2007)1677-1682

[6] Q. He, L. Wang, B. Liu, Parameter estimation for chaotic systems by particle swarln optimization, Chaos, Solitons and Fractals, 34, (2007)654-661

[7] W. D. Chang, Parameter identification of Chen and Ld systems: A differential evolution approach, Chaos, Solitons and Fractals, 32, (2007)1469-1476

[8]Wu S. F., Engelen J. H., BabuSka R. Intelligent flight controller design with fuzzy logic for atmospheric reentry Vehicle. AIAA, Aerospace Sciences Meeting and Exhibit, 2000

[9]Austin K. J., Jacobs P. A., Application of Genetic Algorithms to Hypersonic Flight Control[A]. IFSA World Congress and 20th NAFIPS International Conference Austrilia, 2001, 2428-2433

[10]Elmar M. Wallner, Klaus H. Well, Attitude control of a reentry vehicle with internal dynamics [A], In: AIAA Guidance, Navigation, and Control Conference and Exhibit [C], Monterey: AIAA, 2002-4647

[11]S. F. Wu, C. J. H. Engelen, R. Babuska, Fuzzy logic full-envelope autonomous flight control for an atmospheric re-entry spacecraft [J], Control Engineering Practice, 2003 (11): 11-25 